\title{
The effect of telmisartan on the ventricular systolic function in dogs with experimental supraventricular tachyarrhythmia
}

\author{
Takae KAWAGUCHI 1), Rina HASHIMOTO'), Youko YASUKAWA ${ }^{1)}$, \\ Shusaku YAMADA ${ }^{1}$, , Aritada YOSHIMURA ${ }^{1)}$, Daiki HIRAO ${ }^{1)}$, Takahiro OHMORI') \\ and Ryuji FUKUSHIMA ${ }^{1) *}$
}

${ }^{1)}$ Tokyo University of Agriculture and Technology Animal Medical Center, 3-5-8 Saiwai-cho, Fuchu-shi, Tokyo 183-8509, Japan

J. Vet. Med. Sci.

81(5): 717-722, 2019

doi: 10.1292/jvms.18-0772

Received: 26 December 2018 Accepted: 19 March 2019

Published online in J-STAGE: 8 April 2019
ABSTRACT. Maintaining a good ventricular systolic function is important in the long-term therapy of dogs with supraventricular tachyarrhythmia (SVTA). The objective of this study was to evaluate the inhibitory effect of telmisartan on myocardial injury and the resulting ventricular systolic dysfunction in a canine model of SVTA. A total of 14 dogs were randomly assigned to a Telmisartan (oral telmisartan, $1.0 \mathrm{mg} / \mathrm{kg}$ daily, $\mathrm{n}=7$ ) or a Control (no drug administration, $\mathrm{n=7}$ ) group; the duration of rapid atrial pacing (RAP) was 3 weeks for both groups. The cardiac troponin I (cTnl) concentration in the Control group was significantly increased after 3 weeks compared to that before RAP initiation (baseline), but no significant difference was observed in the Telmisartan group. Moreover, the cTnl concentration at 3 weeks was significantly lower in the Telmisartan group than in the Control group. The left ventricular fractional shortening was significantly decreased at 3 weeks compared to that at baseline in both groups. However, fractional shortening at 3 weeks was significantly higher in the Telmisartan group than in the Control group. The cardiac output values in the Control group were significantly decreased at 3 weeks compared with those at baseline, but no significant difference was observed in the Telmisartan group. This study demonstrates that telmisartan inhibits the reduction in ventricular systolic function and prevents myocardial injury in a canine model of SVTA. Therefore, telmisartan is suggested as a novel treatment for canine SVTA.

KEY WORDS: cardiac troponin I, dog, supraventricular tachyarrhythmia, telmisartan, ventricular systolic function

In dogs, supraventricular tachyarrhythmia (SVTA), such as supraventricular tachycardia, atrial fibrillation, or atrial flutter, represents an arrhythmia observed independently or accompanying heart disease and other systemic disorders [15]. In SVTA, supraventricular impulses are transmitted to the ventricles at a high frequency, leading to an increased ventricular rate [15]. A continued increased ventricular rate results in a reduced ventricular systolic function [23]. Furthermore, if the reduction in ventricular systolic function is sustained for a long period, it may cause severe heart failure [23, 35]. Therefore, it is important to establish treatments for maintaining a stable ventricular systolic function during long-term therapy of dogs with SVTA.

It has been reported that myocardial injury such as degeneration and fibrosis of the myocardial tissue can be caused by activation of the renin-angiotensin-aldosterone system (RAAS) in animals with SVTA and that pathological changes caused by RAAS over-activation play an important role in the development of ventricular systolic dysfunction [30]. Angiotensin-converting enzyme (ACE) inhibitors (ACEIs) and angiotensin II (ANG II) type 1 receptor (AT1) blockers (ARBs) are known as drug groups that control RAAS activity. ACEIs inhibit the activity of the enzyme that converts ANG I to ANG II and reduce the effects of ANG II, which plays a central role in the RAAS, by decreasing ANG II production. However, in humans and dogs, it was demonstrated that the production of ANG II is supported by mast cell-derived chymase in addition to ACE [1,28]. Therefore, it was suggested that the activity of ANG II cannot be completely inhibited by ACEIs alone. For this reason, ARBs have been developed for use in humans to selectively block AT1 receptors and inhibit ANG II activity at the receptor level [31]. Funabiki et al. compared the inhibitory effects of ARBs and ACEIs on myocardial fibrosis in dogs. Their study showed that treatment with either an ARB or an ACEI alone is less effective than the combination therapy. Nevertheless, ARBs are more effective than ACEIs [9]. Ohta et al. reported that ARBs show superior effects compared to ACEIs in preventing cardiac contractile dysfunction in dogs with

*Correspondence to: Fukushima, R.: ryu-ji@cc.tuat.ac.jp

O2019 The Japanese Society of Veterinary Science

This is an open-access article distributed under the terms of the Creative Commons Attribution Non-Commercial No Derivatives (by-nc-nd) License. (CC-BY-NC-ND 4.0: https://creativecommons.org/licenses/by-nc-nd/4.o/) 
tachycardia [19].

Recently, telmisartan, an ARB, was approved and released in several countries for use as a therapeutic drug in animals and is currently used to treat proteinuria in cats with chronic kidney disease (chronic renal failure). Schierok et al. [20] reported that telmisartan was also effective in dogs with renal diseases. A therapeutic effect of telmisartan was also suggested in dogs with chronic kidney disease (chronic renal failure) and its clinical efficacy for the treatment of protein leakage into urine was confirmed [2]. Moreover, telmisartan was reported to exert protective and maintenance effects on ventricular function in a dog model of stunned myocardium $[13,17]$.

The objective of this study was to evaluate the inhibitory effects of telmisartan, an ARB agent approved for animal use, on myocardial injury and the resulting ventricular systolic dysfunction during the development of SVTA in a dog model.

\section{MATERIALS AND METHODS}

This study was conducted in accordance with the Ethical Code for Animal Experiments of the Tokyo University of Agriculture and Technology (Approval number: 27-38).

\section{Animals}

A total of 14 clinically healthy mature beagles ( 8 males and 6 females, 2-5 years old, body weight $9-11 \mathrm{~kg}$ ) were included in this study. Dogs were provided food containing $1.8 \times\left[70 \times(\mathrm{kg} \text { body weight })^{0.75}\right] \mathrm{kcal} / \mathrm{day}$ [5], and water was supplied ad libitum. The dogs were individually housed during the study. The environment was maintained at a temperature of $21 \pm 2{ }^{\circ} \mathrm{C}$ and a relative humidity of $50 \pm 20 \%$, with a $12 \mathrm{hr}: 12 \mathrm{hr}$ light-dark cycle. In addition, the dogs could run freely in a playground for $30 \mathrm{~min}$ every day. SVTA canine models with induced artificial tachycardia were prepared by continuously applying high-frequency electric stimulation to the atrium $[8,23]$. The procedures for developing the SVTA canine models have been described in detail by Ohashi et al. [18] and Gaspo et al. [10]. Animals were pre-anesthetized using a subcutaneous injection of atropine sulfate at $0.05 \mathrm{mg} /$ $\mathrm{kg}$ (Mitsubishi Tanabe Pharma Co., Osaka, Japan) and an intravenous injection of midazolam at $0.2 \mathrm{mg} / \mathrm{kg}$ (Dormicum; Astellas Pharma, Tokyo, Japan). Anesthesia was induced using an intravenous injection of propofol at 5-6 mg/kg (Propofol; Fresenius Kabi, Tokyo, Japan) and isoflurane (Isoflur; DS Pharma Animal Health, Osaka, Japan) was administered to maintain anesthesia via an endotracheal tube (end-tidal isoflurane concentration: 1.3-1.7\%). A thoracotomy was performed at the fifth intercostal space and an atrial stimulation electrode lead (TY216-033; UNIQUE Medical Co., Ltd., Tokyo, Japan) was sutured to the left atrial appendage. The electrode lead was passed through a subcutaneous tunnel and exposed at the back of the body. The chest was then closed, as done routinely. For perioperative pain management, $0.02 \mathrm{mg} / \mathrm{kg}$ of buprenorphine hydrochloride (Lepetan injection, Otsuka Pharmaceutical Co., Ltd., Tokyo, Japan) was intravenously injected pre-operatively and bupivacaine hydrochloride (Marcain; AstraZeneca K. K., Osaka, Japan), and lidocaine (Xylocaine; Aspen Japan, Tokyo, Japan) were administered at the incision area. A meloxicam dose of $0.3 \mathrm{mg} / \mathrm{kg}$ (Metacam 0.5\%; Boehringer Ingelheim Animal Health Japan Co., Ltd., Tokyo, Japan) was administered subcutaneously at 3 days post-surgery. After the operation, the dogs were placed in a jacket with a pocket in which an external cardiac pacemaker (EV4543; Taisho Biomed Instruments Co., Ltd., Tokyo, Japan) was installed. The recovery period after the surgery was 14 days. The 14 dogs were randomly assigned to either a group that did not receive medication during rapid atrial pacing (RAP) (Control group, $n=7$ ) or a group that received telmisartan during RAP (Telmisartan group, $n=7$ ). After the recovery period, the electrode leads were connected to the pacemakers in both groups and RAP was initiated; stimulations of 2 Volts were administered at a frequency of 390 beats per min (bpm). The Telmisartan group was orally administered with telmisartan (Semintra; Boehringer Ingelheim Animal Health Japan Co., Ltd.) at $1.0 \mathrm{mg} / \mathrm{kg}$ once per day (AM 8:00-9:00) during RAP. The period of RAP was 3 weeks for both groups.

\section{Procedures}

We conducted measurements of cardiac troponin I (cTnI) concentration, echocardiography, and blood pressure measurements before starting RAP (Baseline) and 3 weeks after RAP initiation. The examinations after 3 weeks were conducted 1 hr after the temporary pause of RAPs to capture the pathological features of the heart (Fig. 1). All examinations were performed without the use of anesthetics. Furthermore, to avoid the influence of diurnal variations on the measured values, all experimental procedures were conducted at a fixed time (PM 4:00-5:00).

\section{Ventricular rate}

This parameter was calculated per min using an electrocardiogram (D300; Fukuda M-E Kogyo Co., Ltd., Tokyo, Japan). The ventricular rate during RAP was measured right after and 3 weeks after RAP initiation. All examinations were performed without anesthetics.

\section{cTnI concentration}

Blood samples were collected from the jugular vein in a tube with serum-separating medium and centrifuged at 3,000 rpm for $10 \mathrm{~min}$. Measurement of cTnI concentration was performed by a commercial laboratory (FUJIFILM Monolith, Co, Ltd., Tokyo, Japan). Plasma cTnI concentration was measured using a highly sensitive chemiluminescence enzyme immunoassay (ADVIA Centaur CP TnI-ultra, Siemens Healthineers Japan, Tokyo, Japan). It is a 3-site, second-generation sandwich immunoassay employing direct chemiluminometry; the lower and upper limits of cTnI detection were 0.006 and $50 \mathrm{ng} / \mathrm{ml}$. 


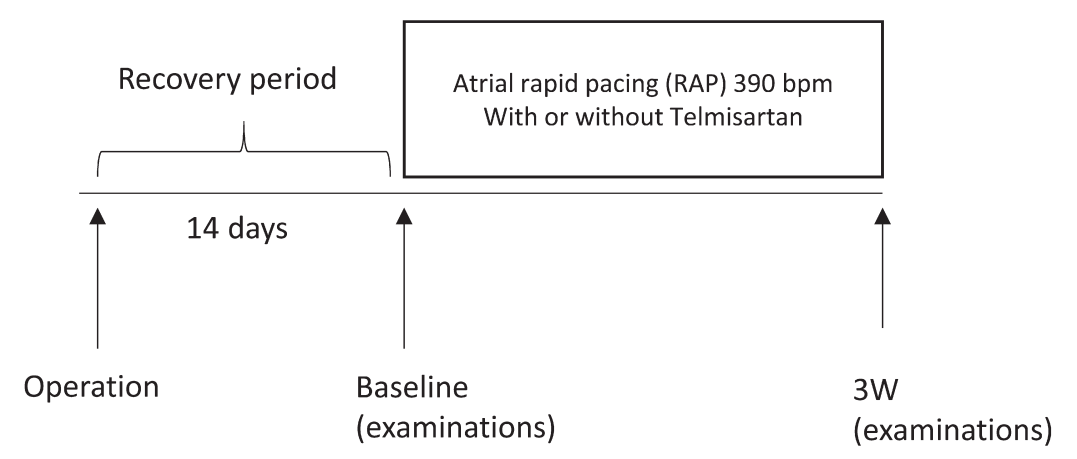

Fig. 1. Schematic outline of study design. Postoperative recovery period was 14 days. Then, measurement of cardiac troponin I concentration, blood pressure measurement, and echocardiography were performed before the initiation of RAP (Baseline) and 3 weeks after the initiation of RAP. Examinations at 3 weeks were carried out $1 \mathrm{hr}$ after RAP was paused. RAP, rapid atrial pacing; bpm, beats per min; W, weeks.

\section{Blood pressure measurements}

The systolic arterial pressure (SAP), mean arterial pressure (MAP), and diastolic arterial pressure (DAP) were measured at the tail-head using a noninvasive oscillometric sphygmomanometer for animals (BP-100D; Fukuda M-E Kogyo Co., Ltd.) with a cuff width of $40-60 \%$ of the circumference of the measured region [4]. Measurements were obtained in triplicates and, when the errors were within $3 \mathrm{mmHg}$, the mean value of the 3 measurements was used.

\section{Echocardiography}

The echocardiography was carried out using an ultrasound unit (Logiq 7; GE Health Care Co., Ltd., Tokyo, Japan). The enddiastolic and end-systolic left-ventricular internal diameter (LVIDd and LVIDs, respectively), and the left-ventricular fractional shortening (FS), an index of the left-ventricular systolic function, were measured using the M-mode on the right parasternal short-axis view at the level of the tendinous cord. Heart rates (HRs) were recorded simultaneously. Using the left parasternal apical 5-chamber view, the ratio between pre-ejection period and ejection time (PEP/ET) was determined from the waveform of the leftventricular outflow tract, wherein the sample volume was set on the closed site of the aortic valve. The PEP was defined as the time interval between the $\mathrm{Q}$ wave and the beginning of the ventricular ejection $[7,16]$. In the same view, cardiac output $(\mathrm{CO})$ and stroke volume (SV) were obtained by measuring the aortic diameter (d) and the velocity time integral (VTI) based on the left-ventricular outflow on pulsed-wave Doppler imaging. The following equations were used: $\mathrm{SV}=(\mathrm{d} / 2)^{2} \times \pi \times \mathrm{VTI}$ and $\mathrm{CO}=\mathrm{SV} \times \mathrm{HR}$ [7]

Echocardiography data were measured over an average of 9 consecutive beats.

\section{Statistical analysis}

Data were expressed as the mean \pm standard deviation. The normal distribution of the measurements was analyzed using the Kolmogorov-Smirnov test. Based on these results, data obtained at baseline and at 3 weeks were statistically analyzed by a paired $t$-test or Wilcoxon signed-rank test for intragroup comparison and by Student's $t$-test or Mann-Whitney $U$-test for intergroup comparison. The statistical analyses were performed using statistical software (BellCurve for Excel; Social Survey Research Information Co., Ltd., Tokyo, Japan), and $P$-values $<0.05$ were considered statistically significant.

\section{RESULTS}

No significant differences between the 2 groups were observed in the baseline measurements of any tested feature.

\section{Ventricular rate}

In the Control group and the Telmisartan group, the ventricular rates were $185 \pm 12 \mathrm{bpm}$ and $187 \pm 8 \mathrm{bpm}$ immediately after stimulation, and $188 \pm 20 \mathrm{bpm}$ and $190 \pm 15 \mathrm{bpm}$ at 3 weeks, respectively. No significant differences were observed either within or between the groups.

\section{cTnI concentration}

The cTnI concentration in the Control group increased significantly at 3 weeks after RAP initiation compared with that at Baseline $(0.025 \pm 0.034 \mathrm{ng} / \mathrm{m} /$ versus $0.159 \pm 0.094 \mathrm{ng} / \mathrm{m} l)(P<0.01)$, but no significant difference was observed in the Telmisartan group $(0.018 \pm 0.029 \mathrm{ng} / \mathrm{m} l$ versus $0.015 \pm 0.011 \mathrm{ng} / \mathrm{m} l)$. The $\mathrm{cTnI}$ concentration at 3 weeks was significantly lower in the Telmisartan group than in the Control group $(P<0.01)$ (Fig. 2).

\section{Blood pressure examination}

The SAP was significantly decreased at 3 weeks compared with that at Baseline in both groups (both $P<0.01$ ). The MAP was significantly decreased in the Telmisartan group at 3 weeks compared with that at Baseline. By contrast, the MAP in the Control 


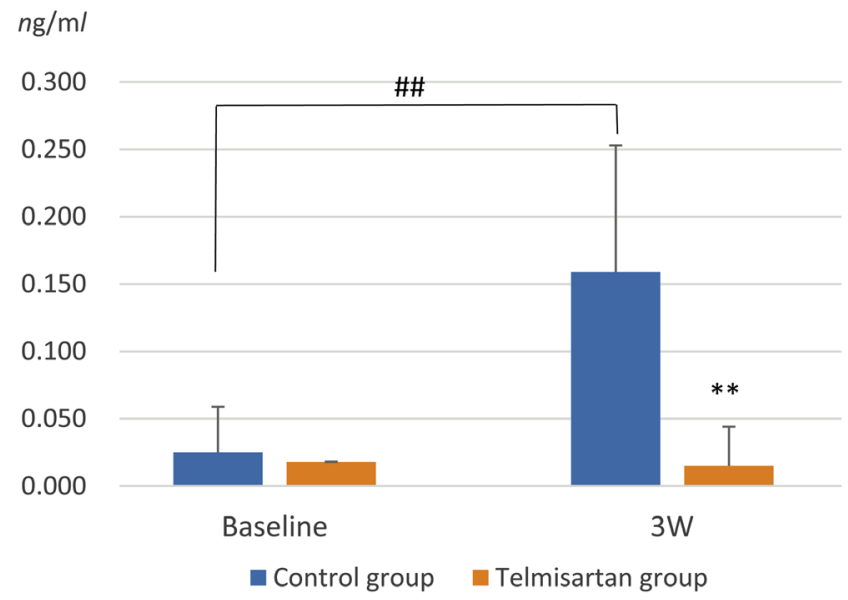

Fig. 2. Changes over time of cardiac troponin I concentration in Control and Telmisartan groups. Baseline, before rapid atrial pacing (RAP); $3 \mathrm{~W}, 3$ weeks after RAP; Control, RAP with no medication; Telmisartan, RAP with telmisartan administration; \#,$P<0.01$ versus Baseline; **, $P<0.01$ versus Control group.

group did not significantly differ between 3 weeks and Baseline. The MAP and DAP values (each $P<0.01$ ) at 3 weeks were significantly lower in the Telmisartan group than in the Control group (Table 1).

\section{Echocardiography}

The LVIDs was significantly larger in the Control group than in the Telmisartan group at 3 weeks $(P<0.01)$. No significant change was found in the LVIDd of the Control group. By contrast, in the Telmisartan group, a significantly smaller LVIDd was observed at 3 weeks compared with that at Baseline $(P<0.01)$. A significantly larger LVIDs was observed in the Control group at 3 weeks compared to that at Baseline $(P<0.01)$ (Table 2). FS decreased significantly after RAP compared with that at Baseline in both groups. However, FS was significantly greater at 3 weeks in the Telmisartan group than in the Control group $(P<0.01)$ (Table 2$)$. The ratio PEP/ET was significantly increased at 3 weeks after RAP initiation compared with that at Baseline, in both animal groups. However, the PEP/ET ratio at 3 weeks was significantly lower in the Telmisartan group compared to that in the Control group $(P<0.01)$ (Table 2$)$. The $\mathrm{CO}$ and $\mathrm{SV}$ values in the Control group were significantly decreased at 3 weeks compared with those at Baseline (both $P<0.01$ ), but no significant difference was observed in these values in the Telmisartan group. No significant difference was found in the HR either within or between the groups (Table 2).
Table 1. Value of blood pressure measurement parameter in Control and Telmisartan groups

\begin{tabular}{llcc}
\hline Parameter & Group & Baseline & $3 \mathrm{~W}$ \\
\hline SAP & Control & $147 \pm 19$ & $125 \pm 17^{\mathrm{a})}$ \\
& Telmisartan & $143 \pm 6$ & $107 \pm 12^{\mathrm{a})}$ \\
MAP & Control & $110 \pm 15$ & $102 \pm 16$ \\
& Telmisartan & $101 \pm 9$ & $73 \pm 14^{\mathrm{a}, \mathrm{b})}$ \\
\multirow{3}{*}{ DAP } & Control & $92 \pm 19$ & $91 \pm 21$ \\
& Telmisartan & $82 \pm 13$ & $60 \pm 14^{\mathrm{b})}$ \\
\hline
\end{tabular}

All values are expressed as mean \pm standard deviation. SAP, systolic arterial pressure; MAP, mean arterial pressure; DAP, diastolic arterial pressure; Baseline, before rapid atrial pacing (RAP); $3 \mathrm{~W}, 3$ weeks after RAP; Control, RAP with no medication; Telmisartan, RAP with telmisartan administration. a) $P<0.01$ versus Baseline, b) $P<0.01$ versus Control group.

Table 2. Values of echocardiographic parameters in Control and Telmisartan groups

\begin{tabular}{llll}
\hline Parameter & \multicolumn{1}{c}{ Group } & \multicolumn{1}{c}{ Baseline } & \multicolumn{1}{c}{$3 \mathrm{~W}$} \\
\hline HR & Control & $128 \pm 33$ & $122 \pm 30$ \\
& Telmisartan & $108 \pm 22$ & $120 \pm 22$ \\
LVIDd & Control & $31.0 \pm 2.2$ & $29.2 \pm 3.5$ \\
& Telmisartan & $29.0 \pm 3.8$ & $26.9 \pm 2.0^{\text {a) }}$ \\
LVIDs & Control & $19.2 \pm 1.9$ & $22.5 \pm 4.0^{\mathrm{a})}$ \\
& Telmisartan & $17.1 \pm 1.8$ & $18.6 \pm 1.5^{\mathrm{b})}$ \\
FS & Control & $38.2 \pm 3.8$ & $23.4 \pm 5.6^{\mathrm{a})}$ \\
& Telmisartan & $38.2 \pm 5.1$ & $31.5 \pm 6.1^{\mathrm{a}, \mathrm{b})}$ \\
PEP/ET & Control & $0.13 \pm 0.03$ & $\left.0.33 \pm 0.07^{\mathrm{a}}\right)$ \\
& Telmisartan & $0.12 \pm 0.03$ & $\left.0.19 \pm 0.06^{\mathrm{a}}, \mathrm{b}\right)$ \\
SV & Control & $17.0 \pm 5.8$ & $10.9 \pm 3.4^{\mathrm{a})}$ \\
& Telmisartan & $15.2 \pm 5.0$ & $13.7 \pm 4.9$ \\
CO & Control & $1.99 \pm 0.48$ & $1.31 \pm 0.34^{\mathrm{a})}$ \\
& Telmisartan & $1.72 \pm 0.51$ & $1.55 \pm 0.68$ \\
\hline
\end{tabular}

All values are expressed as mean \pm standard deviation. HR, heart rate; LVIDd, end-diastolic left ventricle internal dimension; LVIDs, end-systolic left ventricle internal dimension; FS, fractional shortening; PEP/ET, pre-ejection period/ejection time; SV, stroke volume; $\mathrm{CO}$, cardiac output; Baseline, before rapid atrial pacing (RAP); 3 W, 3 weeks after RAP; Control, RAP with no medication; Telmisartan, RAP with telmisartan administration; a) $P<0.01$ vs Baseline, b) $P<0.01$ vs Control.

\section{DISCUSSION}

In clinical cases, the ventricular rate of dogs with SVTA often increases to values over $200 \mathrm{bpm}$, and in some cases, this rate exceeds $300 \mathrm{bpm}$ [15]. In this study, the ventricular rates of the Telmisartan group and the Control group during RAP were over $250 \mathrm{bpm}$, which means that the RAP given was adequate for the establishment of the SVTA model. Moreover, the ventricular rates observed during and after RAP were similar in the Telmisartan group compared with those in the Control group. Therefore, the cardioprotective effect of telmisartan observed in this study does not seem to result from changes in ventricular rate.

The cTnI level is widely utilized as a biomarker for the diagnosis of myocardial infarction in humans [26]. cTnI is a myocardial intracellular protein that is released by damaged and destroyed cardiomyocytes, resulting in greatly elevated concentrations in the blood [32]. Increased levels of cTnI have been reported in dogs with mitral insufficiency [27], filariasis [3], atrioventricular block [29], cardiac hemangiosarcoma [22], and administration of doxorubicin [25]. Therefore, it has been concluded that a high cTnI 
concentration in the blood is not only caused by infarct necrosis of the cardiomyocytes, but also by a variety of heart lesions that induce myocardial injury [11].

In previous studies, myocardial fibrosis formation of the entire ventricle was confirmed microscopically in an animal model that involved high-frequency stimulation of the heart $[6,12]$. Fibrosis of the ventricular muscle tissue is formed as a scar to replace necrotic cardiomyocytes after a myocardial injury [6]. It has also been shown that injury-associated features such as cellular elongation, myofibril misalignment, sarcomere disassembly, and disappearance of cardiomyocytes occur before the onset of myocardial fibrosis [23]. One of these factors that precede fibrosis is a tachycardia-induced reduction in coronary blood flow, i.e., myocardial ischemia [14]. Furthermore, a direct RAAS involvement in the fibrosis formation has already been demonstrated. Stimulation of AT1 receptors by ANG II upregulates the expression of transforming growth factor- $\beta 1$ in the cardiac tissue, stimulates collagen accumulation, inhibits the activity of enzymes that degrade the extracellular matrix, enhances the proliferation of fibroblasts, and induces inflammatory responses, all of which may be responsible for myocardial fibrosis [6, 21]. In the present study, we could not visualize the development of myocardial fibrosis and the preceding myocardial injury because we did not perform histopathological examinations. However, an increase in cTnI, a biomarker of myocardial injury, was observed after RAP in the Control group, suggesting the occurrence of an injury in the canine ventricular muscles, as reported previously [6, 12]. By contrast, no increase in cTnI was observed after RAP in the Telmisartan group. Telmisartan has been shown to ameliorate the reduced blood flow in coronary arteries via activation of AT1 receptors [34]. It was suggested that telmisartan protects myocardial cells against injury by alleviating myocardial ischemia. This is in agreement with the results of the present study; however, a direct inhibition of AT1 receptor-mediated responses, including activation of extracellular matrix-degrading enzymes and proliferation of myocardial fibroblasts, may also have contributed to the inhibitory effects of telmisartan on RAP-induced fibrosis in the current study. Besides AT1 receptor activation, ANG II also stimulates AT2 receptors that act antagonistically to AT1 receptor-mediated responses $[24,33]$. ARBs cause an elevation in ANG II concentration in the blood via a feedback mechanism involving the blockade of AT1 receptors, resulting in the predominant activity of AT2 receptors [24, 33]. Therefore, administration of telmisartan might have suppressed myocardial injury by blocking the AT1 receptor-mediated responses and enhancing the AT2 receptormediated responses.

In the present study, the ventricular systolic function was assessed by echocardiography. Generally, a reduced FS is considered to be caused by a decreased preload, an enhanced afterload, or a reduced systolic function of the left ventricle [7]. In the present study, the FS decreased after atrial stimulation in both the Control and the Telmisartan group. However, the reduction in FS was less marked in the Telmisartan group than in the Control group. The parameter FS in both groups largely depended on LVIDs variations. A significant increase in LVIDs was observed after RAP in the Control group but not in the Telmisartan group. These findings suggest that a myocardial injury indicated by an increase in cTnI concentration in the blood is directly associated with the changes in LVIDs and the decrease in left-ventricular contractility in the Control group. Since the myocardial injury was controlled in the Telmisartan group, it is considered that the cardiac contractility was maintained and the LVIDs were largely maintained as compared with the Control group. Moreover, the decrease in blood pressure observed in the Telmisartan group in this study indicated a decrease in afterload probably due to the blockade of the AT1 receptor effect, which may also have contributed to the increase in FS. Our data suggest that telmisartan suppresses the decrease in FS by the interaction of two factors: suppression of the myocardial injury and reduction of the afterload. In the present study, the ratio PEP/ET was increased in both the Control and the Telmisartan group, although the change was less marked in the Telmisartan group. The PEP/ET ratio was also utilized as an index to evaluate the ventricular systolic function with an increase or decrease in this index representing a reduced or enhanced systolic function, respectively [7]. The effect of telmisartan on PEP/ET is considered to be held by maintenance of ventricular contractility, similarly to the interpretation of its effect on FS. Likewise, SV and CO decreased in the Control group after RAP stimulation, but both parameters were maintained in the Telmisartan group at baseline levels due to the preventive effects of telmisartan on the ventricular systolic dysfunction.

A limitation of the present study is the difference in pathophysiology between our animal model of SVTA and clinical cases. The SVTA model in this study was produced by applying RAP in healthy young beagles. In clinical cases, the dogs with SVTA are older than the model dogs and often have valvular diseases, such as mitral and tricuspid regurgitation [15]. Therefore, prospective studies are necessary to be conducted in clinical cases on the basis of the results from our study.

In conclusion, the present study demonstrated that telmisartan suppressed myocardial injury and reduced ventricular systolic dysfunction in a canine model of SVTA. Therefore, telmisartan may be a promising novel drug for SVTA in dogs, provided that blood pressure reduction is carefully monitored.

\section{REFERENCES}

1. Akasu, M., Urata, H., Kinoshita, A., Sasaguri, M., Ideishi, M. and Arakawa, K. 1998. Differences in tissue angiotensin II-forming pathways by species and organs in vitro. Hypertension 32: 514-520. [Medline] [CrossRef]

2. Bugbee, A. C., Coleman, A. E., Wang, A., Woolcock, A. D. and Brown, S. A. 2014. Telmisartan treatment of refractory proteinuria in a dog. J. Vet. Intern. Med. 28: 1871-1874. [Medline] [CrossRef]

3. Carretón, E., Morchón, R., Simón, F., Juste, M. C., González-Miguel, J. and Montoya-Alonso, J. A. 2014. Evaluation of cardiopulmonary biomarkers during classic adulticide treatment versus the American Heartworm Society recommended treatment protocol in dogs infected by Dirofilaria immitis. Vet. Parasitol. 206: 55-59. [Medline] [CrossRef]

4. Coulter, D. B. and Keith, J. C. Jr. 1984. Blood pressures obtained by indirect measurement in conscious dogs. J. Am. Vet. Med. Assoc. 184: 1375-1378. [Medline] 
5. Crane, W. S., Griffin, W. R. and Messent, R. P. 2000. Introduction to commercial pet foods. pp. 111-126. In: Small Animal Clinical Nutrition, 4th ed. (Hand, S. M., Thatcher, D. C., Remillard, L. R. and Roudebush, P. eds.), Mark Morris Institute, Kansas.

6. Dzeshka, M. S., Lip, G. Y., Snezhitskiy, V. and Shantsila, E. 2015. Cardiac Fibrosis in Patients With Atrial Fibrillation: Mechanisms and Clinical Implications. J. Am. Coll. Cardiol. 66: 943-959. [Medline] [CrossRef]

7. Feigenbaum, H. 1986. Echocardiographic evaluation of cardiac chambers. pp. 127-187. In: Echocardiography, 4th ed. (Feigen Baum, H. ed.), Lea \& Febiger, Philadelphia.

8. Fukushima, R., Tanaka, R., Matsumoto, H., Machida, N., Hirose, H., Yamane, Y. and Koyama, H. 2010. Effects of enfonidipine hydrochloride in dogs with experimental supraventricular tachyarrhythmia. J. Vet. Med. Sci. 72: 833-838. [Medline] [CrossRef]

9. Funabiki, K., Onishi, K., Dohi, K., Koji, T., Imanaka-Yoshida, K., Ito, M., Wada, H., Isaka, N., Nobori, T. and Nakano, T. 2004. Combined angiotensin receptor blocker and ACE inhibitor on myocardial fibrosis and left ventricular stiffness in dogs with heart failure. Am. J. Physiol. Heart Circ. Physiol. 287: H2487-H2492. [Medline] [CrossRef]

10. Gaspo, R., Bosch, R. F., Talajic, M. and Nattel, S. 1997. Functional mechanisms underlying tachycardia-induced sustained atrial fibrillation in a chronic dog model. Circulation 96: 4027-4035. [Medline] [CrossRef]

11. Giannitsis, E. and Katus, H. A. 2013. Cardiac troponin level elevations not related to acute coronary syndromes. Nat. Rev. Cardiol. 10: 623-634. [Medline] [CrossRef]

12. Healey, J. S., Morillo, C. A. and Connolly, S. J. 2005. Role of the renin-angiotensin-aldosterone system in atrial fibrillation and cardiac remodeling. Curr. Opin. Cardiol. 20: 31-37. [Medline]

13. Izumi, H., Nakai, T., Kano, S., Hoshi, K. and Ichihara, K. 1996. Effects of BIBR-277, an angiotensin II type 1 receptor antagonist, on stunned myocardium in dogs. Coron. Artery Dis. 7: 775-779. [Medline] [CrossRef]

14. Jalowy, A., Schulz, R. and Heusch, G. 1999. AT1 receptor blockade in experimental myocardial ischemia/reperfusion. J. Am. Soc. Nephrol. 10 Suppl 11: S129-S136. [Medline]

15. Kittleson, D. M. 1988. Diagnosis and treatment of arrhythmias. pp. 449-494. In: Small Animal Cardio Vascular Medicine, 1st ed. (Kittleson, M. D. and Kienle, R. P. eds.), Mosby, St. Louis.

16. Matsuno, Y., Morioka, S., Murakami, Y., Kobayashi, S. and Moriyama, K. 1988. Mechanism of prolongation of pre-ejection period in the hypertrophied left ventricle with normal systolic function in unanesthetized hypertensive dogs. Clin. Cardiol. 11: 702-706. [Medline] [CrossRef]

17. Nakai, T., Satoh, K., Kosugi, T., Hoshi, K. and Ichihara, K. 1999. Participation of angiotensin II and bradykinin in contractile function in dog stunned myocardium. Eur. J. Pharmacol. 382: 187-196. [Medline] [CrossRef]

18. Ohashi, N., Mitamura, H., Tanimoto, K., Fukuda, Y., Kinebuchi, O., Kurita, Y., Shiroshita-Takeshita, A., Miyoshi, S., Hara, M., Takatsuki, S. and Ogawa, S. 2004. A comparison between calcium channel blocking drugs with different potencies for T- and L-type channels in preventing atrial electrical remodeling. J. Cardiovasc. Pharmacol. 44: 386-392. [Medline] [CrossRef]

19. Ohta, T., Hasebe, N., Tsuji, S., Izawa, K., Jin, Y. T., Kido, S., Natori, S., Sato, M. and Kikuchi, K. 2004. Unequal effects of renin-angiotensin system inhibitors in acute cardiac dysfunction induced by isoproterenol. Am. J. Physiol. Heart Circ. Physiol. 287: H2914-H2921. [Medline] [CrossRef]

20. Schierok, H., Pairet, M., Hauel, N. and Wienen, W. 2001. Effects of telmisartan on renal excretory function in conscious dogs. J. Int. Med. Res. 29: 131-139. [Medline] [CrossRef]

21. Segura, A. M., Frazier, O. H. and Buja, L. M. 2014. Fibrosis and heart failure. Heart Fail. Rev. 19: 173-185. [Medline] [CrossRef]

22. Shaw, S. P., Rozanski, E. A. and Rush, J. E. 2004. Cardiac troponins I and T in dogs with pericardial effusion. J. Vet. Intern. Med. 18: 322-324. [Medline] [CrossRef]

23. Shinbane, J. S., Wood, M. A., Jensen, D. N., Ellenbogen, K. A., Fitzpatrick, A. P. and Scheinman, M. M. 1997. Tachycardia-induced cardiomyopathy: a review of animal models and clinical studies. J. Am. Coll. Cardiol. 29: 709-715. [Medline] [CrossRef]

24. Siragy, H. 1999. Angiotensin II receptor blockers: review of the binding characteristics. Am. J. Cardiol. 84 10A: 3S-8S. [Medline] [CrossRef]

25. Surachetpong, S. D., Teewasutrakul, P. and Rungsipipat, A. 2016. Serial measurements of cardiac troponin I (cTnI) in dogs treated with doxorubicin. Jpn. J. Vet. Res. 64: 221-233. [Medline]

26. Thygesen, K., Alpert, J. S., Jaffe, A. S., Chaitman, B. R., Bax, J. J., Morrow, D. A., White H. D., Executive Group on behalf of the Joint European Society of Cardiology (ESC)/American College of Cardiology (ACC)/American Heart Association (AHA)/World Heart Federation (WHF) Task Force for the Universal Definition of Myocardial Infarction. 2018. Fourth Universal Definition of Myocardial Infarction (2018). J. Am. Coll. Cardiol. 72: 2231-2264. [Medline] [CrossRef]

27. Wall, L., Mohr, A., Ripoli, F. L., Schulze, N., Penter, C. D., Hungerbuehler, S., Bach, J. P., Lucas, K. and Nolte, I. 2018. Clinical use of submaximal treadmill exercise testing and assessments of cardiac biomarkers NT-proBNP and cTnI in dogs with presymptomatic mitral regurgitation. PLoS One 13: e0199023. [Medline] [CrossRef]

28. Wei, C. C., Meng, Q. C., Palmer, R., Hageman, G. R., Durand, J., Bradley, W. E., Farrell, D. M., Hankes, G. H., Oparil, S. and Dell’Italia, L. J. 1999. Evidence for angiotensin-converting enzyme- and chymase-mediated angiotensin II formation in the interstitial fluid space of the dog heart in vivo. Circulation 99: 2583-2589. [Medline] [CrossRef]

29. Wiedemann, N., Hildebrandt, N., Wurtinger, G., Henrich, E., Hassdenteufel, E. and Schneider, M. 2017. Follow-up of troponin I concentration in dogs with atrioventricular block and dual-chamber pacing in a case-matched study. J. Vet. Cardiol. 19: 247-255. [Medline] [CrossRef]

30. Wijesurendra, R. S. and Casadei, B. 2015. Atrial fibrillation: effects beyond the atrium? Cardiovasc. Res. 105: 238-247. [Medline] [CrossRef]

31. Wong, P. C., Chiu, A. T., Price, W. A., Thoolen, M. J., Carini, D. J., Johnson, A. L., Taber, R. I. and Timmermans, P. B. 1988. Nonpeptide angiotensin II receptor antagonists. I. Pharmacological characterization of 2-n-butyl-4-chloro-1-(2-chlorobenzyl)imidazole-5-acetic acid, sodium salt (S-8307). J. Pharmacol. Exp. Ther. 247: 1-7. [Medline]

32. Wu, A. H. and Feng, Y. J. 1998. Biochemical differences between cTnT and cTnI and their significance for diagnosis of acute coronary syndromes. Eur. Heart J. 19 Suppl N: N25-N29. [Medline]

33. Yamane, T., Fujii, Y., Orito, K., Osamura, K., Kanai, T. and Wakao, Y. 2008. Comparison of the effects of candesartan cilexetil and enalapril maleate on right ventricular myocardial remodeling in dogs with experimentally induced pulmonary stenosis. Am. J. Vet. Res. 69: 1574-1579. [Medline] [CrossRef]

34. Zhang, C., Knudson, J. D., Setty, S., Araiza, A., Dincer, U. D., Kuo, L. and Tune, J. D. 2005. Coronary arteriolar vasoconstriction to angiotensin II is augmented in prediabetic metabolic syndrome via activation of AT1 receptors. Am. J. Physiol. Heart Circ. Physiol. 288: H2154-H2162. [Medline] [CrossRef]

35. Zupan, I., Rakovec, P., Budihna, N., Brecelj, A. and Kozelj, M. 1996. Tachycardia induced cardiomyopathy in dogs; relation between chronic ventricular tachycardia. Int. J. Cardiol. 56: 75-81. [Medline] [CrossRef] 